\title{
Análisis diferencial técnico-económico de los sistemas productivos de guajolotes en el Estado de México
}

\author{
Gabriela Rodríguez-Licea*, Crhistian Carrillo-Juárez*, Juvencio Hernández-Martínez* y Mercedes Borja Bravo**
}

Recepción: 25 de mayo de 2015

Aceptación: 17 de marzo de 2016

*Universidad Autónoma del Estado de México, México. **Instituto Nacional de Investigaciones Forestales, Agrícolas y Pecuarias, México.

Correos electrónicos: gabyrl1972@hotmail.com; cris_jucarr@hotmail.com; jhmartinez1412@gmail.com; mercedes@inifap.gob.mx

Se agradecen los comentarios de los árbitros de la revista.

(c) $\$=$
Resumen. Se determina la eficiencia técnicoeconómica de la meleagricultura mexiquense a través de un análisis diferencial de los sistemas productivos utilizados en unidades avícolas productoras de guajolotes, que se realizó durante el periodo de engorda de 2011. Después de la estimación de los óptimos técnico y económico y de las funciones de producción, se encontró evidencia empírica de que, dado el grado de especialización y nivel de integración vertical y horizontal, las unidades avícolas tecnificadas son más eficientes que las semitecnificadas y las de traspatio. Los resultados coinciden con otras investigaciones realizadas para diferentes regiones del país.

Palabras clave: óptimos técnico, óptimo económico, función de producción.
Technical-Economic Differential Analysis of the Turkey Productive Systems in the State of Mexico

Abstract. The aim of this work was to determine the technical-productive efficiency of the meleagriculture (a type of poultry) in the State of Mexico, through a differential analysis of the production systems used in poultry units (turkey producers), quantified during the fattening period in 2011. After estimating the production functions and technical and economic optimum; empirical evidence showed that, given the degree of specialization and vertical and horizontal integration level, the industrial systems are more efficient in relationship with the semi-industrial and backyard poultry; results coincide with other researches held in different regions of the country.

Key words: technical optimum, economic optimum, production function approach.

\section{Introducción}

La meleagricultura o producción de guajolote (Meleagris gallopavo) es una de las actividades económicas con mayor tradición en México. No obstante, su participación en el sector pecuario no ha sido superior a $0.4 \%$, lo que ha propiciado que $80 \%$ del mercado interno sea cubierto con importaciones (Financiera Rural, 2012). Un factor que pudiera explicar la situación, es la eficiencia técnicoeconómica de los sistemas de producción meleagrícolas, ya que al ser un determinante de la productividad ante variaciones en los precios de los insumos y del producto, o ante cambios en la demanda, la producción podría verse afectada. Aunado a lo anterior, la diferencia en la eficiencia técnico-económica de los tres sistemas meleagrícolas que imperan en el país (de traspatio, semitecnificado y tecnificado) y su distribución espacial ha traído por consecuencia que $79.48 \%$ de la producción de guajolote en pie y $81.85 \%$ de carne en canal se concentre en seis entidades federativas: Chihuahua, Yucatán, Estado de México, Puebla, Tabasco y Guerrero (Siacon, 2013).

El crecimiento de la meleagricultura en $1.10 \%$ registrado en el Estado de México de 2002 a 2012 llevó a esta entidad a generar en promedio 3247 ton de guajolote en pie y 
2312 ton de carne en canal al año y, por consecuencia, a ocupar la tercera posición a nivel nacional. A pesar de esto, la meleagricultura mexiquense se ha caracterizado por la dispersión y concentración de la producción, y por la diferencia en los rendimientos obtenidos de guajolote en pie y de carne en canal, ya que, Atlacomulco, Zumpango y Toluca aportaron en conjunto $69.34 \%$ de guajolote en pie y $68.88 \%$ de carne en canal (cuadro 1), mientras que los guajolotes con mayor peso vivo en pie y de mayor peso en canal se obtuvieron en Valle de Bravo, 10.670 y 7.522 $\mathrm{kg}$ respectivamente (SIAP, 2012).

El panorama planteado de la meleagricultura mexiquense deja ver que los municipios que generan mayor producción no son los que obtienen los rendimientos más altos de guajolote en pie y de carne en canal, lo que hace suponer que en ellos probablemente se encuentran unidades meleagrícolas que no implementan sistemas de producción eficientes que les permitan tomar decisiones correctas sobre el uso de los factores de producción y por consecuencia obtener buenos rendimientos y ser más productivos.

Bajo este supuesto, se tiene por objetivo realizar un análisis espacial y diferencial técnico-económico de los sistemas productivos de guajolotes en el Estado de México que permita determinar si, dada su distribución geográfica y el uso de los factores productivos, los sistemas tecnificados obtienen mayor eficiencia técnico-económica que los semitecnificados y de traspatio, la cual es medida por los óptimos técnico y económico.

\section{Metodología}

\section{1. Determinación de variables}

Para el análisis diferencial se realizó una investigación de campo en la que se aplicaron encuestas en unidades avícolas dedicadas a la meleagricultura que operan bajo sistemas de

Cuadro 1. Estado de México: producción meleagrícola promedio anual (2002-2012).

\begin{tabular}{|c|c|c|c|c|c|c|}
\hline \multirow{3}{*}{ Municipio } & \multicolumn{3}{|c|}{ Guajolote en pie } & \multicolumn{3}{|c|}{ Carne en canal } \\
\hline & \multirow{2}{*}{$\begin{array}{l}\text { Peso vivo } \\
(\mathrm{kg})\end{array}$} & \multicolumn{2}{|c|}{ Producción } & \multirow{2}{*}{$\begin{array}{l}\text { Peso } \\
(\mathrm{kg})\end{array}$} & \multicolumn{2}{|c|}{ Producción } \\
\hline & & ton & $\%$ & & ton & $\%$ \\
\hline Toluca & 7.790 & 470 & 14.48 & 5.945 & 333 & 14.38 \\
\hline Zumpango & 7.499 & 574 & 17.67 & 5.448 & 415 & 17.95 \\
\hline Texcoco & 9.973 & 318 & 9.80 & 7.223 & 225 & 9.72 \\
\hline Tejupilco & 6.498 & 120 & 3.70 & 5.108 & 89 & 3.87 \\
\hline Atlacomulco & 7.249 & 1208 & 37.19 & 4.938 & 845 & 36.55 \\
\hline Coatepec Harinas & 8.913 & 67 & 2.07 & 6.240 & 48 & 2.08 \\
\hline Valle de Bravo & 10.670 & 272 & 8.38 & 7.522 & 204 & 8.84 \\
\hline Jilotepec & 6.750 & 218 & 6.71 & 4.791 & 153 & 6.61 \\
\hline Estatal & 7.698 & 3247 & 100.00 & 5.945 & 2312 & 100.00 \\
\hline
\end{tabular}

producción tecnificados, semitecnificados y de traspatio, ubicadas en ocho municipios del Estado de México (cuadro 1), las cuales fueron previamente identificadas con base en la información reportada por INEGI (2007) y Sagarpa (2012). A partir de un universo de 350 unidades avícolas se determinó un tamaño muestral proporcional regional aplicando la siguiente fórmula:

$\frac{\left[N * Z_{\alpha}^{2} * p * q\right]}{\left[d_{2}(N-1)+Z_{\alpha}^{2} * p * q\right]}$

Donde $N$ = inventario regional de guajolotes, $Z_{\alpha}=1.962$ con una seguridad de $95 \%, p=$ proporción esperada de $5 \%$ (0.05), y $q=1-p=1-0.05=0.95$. El número de encuestas arrojado fue de 339 que se aplican durante las 28 semanas del periodo de engorda de 2011: Toluca 40, Zumpango 71, Texcoco 27, Tejupilco 13, Atlacomulco 130, Coatepec Harinas 6, Valle de Bravo 29 y Jilotepec 33.

Para elaborar las encuestas y realizar el análisis diferencial de los sistemas productivos se consideraron los siguientes aspectos técnico-económicos: origen, raza, etapa productiva y tamaño de las parvadas; infraestructura, comederos y bebederos de las unidades meleagrícolas; tipo, consumo y costo del alimento; programas de producción, vacunación, desparasitación y limpieza; conversión alimenticia y ganancia de peso; tipo de comercialización, cobertura de mercado y precios de venta. Con la información obtenida en campo se realizó un análisis de frecuencia regional y se estimó un promedio estatal.

\section{2. Análisis diferencial de la eficiencia técnico- económica}

\section{2. 1. Eficiencia técnica}

Con base en la teoría de la producción, la cual refiere que, ante variaciones en los costos de producción una empresa puede tomar la decisión de combinar los factores productivos (trabajo, capital y materias primas) para hacer más eficiente la producción (Pindyck y Rubinfeld, 2009), la meleagricultura puede definirse como la transformación de alimento en guajolote en pie, ya que al ser el alimento un insumo variable y representar en promedio $70 \%$ de los costos de producción su consumo se debe optimizar para hacer más eficiente la producción, aunque la 
relación insumo-producto depende del sistema productivo que opere en cada unidad meleagrícola. En relación con lo anterior, la eficiencia técnica se determinó a través de la función del nivel máximo de producción que puede obtener una empresa con cada combinación de factores (Pindyck y Rubinfeld, 2009), la cual para la meleagricultura sería el peso vivo máximo óptimo que alcanza el guajolote ante la combinación de ingredientes que recibe a través del alimento. Matemáticamente la expresión se puede expresar como:

$P_{g}=f(C A, T)$

Donde, $P_{g}$ es el peso vivo máximo óptimo del guajolote (en $\mathrm{kg}$ ), $C A$ el consumo de alimento (en $\mathrm{kg}$ ) y $T$ la tecnología, entendida como el conocimiento sobre los métodos que podrían aplicarse para transformar los factores productivos en productos (Pindyck y Rubinfeld, 2009) o, dicho en otras palabras, el sistema de producción que implementa cada unidad meleagrícola para transformar el alimento en guajolote en pie.

Por otro lado, dado que en el corto plazo las unidades meleagrícolas difícilmente podrían cambiar sus sistemas de producción, a través de la función de producción se determinó la productividad, entendida como la conversión alimenticia o cantidad de alimento que deberá consumir un guajolote en cada fase productiva para alcanzar el peso vivo máximo óptimo de venta en pie. Las fases productivas del proceso de engorda de los guajolotes expresadas en semanas son: a) iniciación, 0 a 4 b) crecimiento, 5 a 10 c) engorda, 15 a 17 y d) finalización, 17 en adelante.

A partir de la productividad total $(P T)$ o cantidad de bienes producidos por el total de factores utilizados (Pindyck y Rubinfeld, 2009), que se traduce como la ganancia de peso máxima óptima del guajolote obtenida del consumo total de alimento, se calculó la productividad media $(P M e)$ o peso vivo promedio de guajolote obtenido por $\mathrm{kg}$ de alimento consumido, y la productividad marginal $(P M g)$ o pérdida de peso por $\mathrm{kg}$ extra de alimento consumido, expresadas matemáticamente como:

\section{$P T=$ Peso máximo del guajolote por consumo total de alimento}

$P M_{e}=\frac{\text { Peso máximo del guajolote }}{\text { Consumo de alimento }}$

$P M_{g}=\frac{\Delta \text { peso máximo del guajolote }}{\Delta \text { consumo de alimento }}$
Donde, la ganancia de peso y el consumo de alimento están expresados en $\mathrm{kg}$. Una vez obtenidas $P T, P M e$ y $P M g$ su representación gráfica e interpretación permitió identificar el comportamiento de los sistemas en cada una de las tres etapas: a) dado que el guajolote se encuentra en fase de iniciación, crecimiento y engorda, se espera que la $P T$ sea máxima, es decir, que la ganancia de peso sea mayor al aumentar el consumo de alimento. $P M e$ es creciente pero menor a $P M g$ $(P M g>P M e) . b)$ Se espera que el guajolote alcance el peso vivo máximo óptimo para venta que en promedio es de $12 \mathrm{~kg}$, y por consecuencia que la unidad meleagrícola obtenga la eficiencia técnica. $P M e$ deberá ser decreciente y $P M g>0$; por lo tanto, se espera que $P M g<P M e . c$ ) Es la fase de finalización, por lo que ante el consumo adicional de alimento se espera que el guajolote baje de peso o, en el mejor de los casos, mantenga su peso. $P M g$ es negativa $(P M g<0)$ y $P M e$ se mantiene positiva, pero con pendiente negativa.

De este modo, a partir de la relación entre $P T, P M e$ y $P M g$ se identificó para cada sistema meleagrícola el tipo de rendimientos a escala o tasa a la que aumenta la producción cuando se incrementan los factores de producción. Ante la aplicación de economías de escala, en el largo plazo se podrían obtener los siguientes rendimientos: a) crecientes, la producción se duplica con creces cuando se duplican los factores (la ganancia de peso se duplica proporcionalmente cuando se duplica el consumo de alimento); $b$ ) constantes, la producción se duplica cuando se duplican los factores (la ganancia de peso duplica cuando se duplica el consumo de alimento); c) decrecientes, la producción no llega a duplicarse cuando se duplican los factores (la ganancia de peso no se duplica cuando se duplica el consumo de alimento) (Pindyck y Rubinfeld, 2009).

\section{2. 2. Eficiencia económica}

El factor determinante de la eficiencia económica en una empresa es el costo, el cual depende de la tecnología y el precio de los insumos y puede variar en función de la producción. Sin embargo, al ser obtenido el costo total a partir de los costos fijos $(C F)$ o independientes de la producción y de los costos variables $(\mathrm{CV})$ o dependientes de la producción, éste podría no reflejar la eficiencia económica; por lo que, es necesario estimar para $C F$ y $C V$ el costo medio $(\mathrm{CMe})$ o costo total de la empresa dividido por su nivel de producción y el costo marginal $(C M g)$ o variación en el costo cuando se produce una unidad más (Pindyck y Rubinfeld, 2009).

Con base en lo anterior, y partiendo del supuesto que los sistemas meleagrícolas manejan periodos de engorda de corto plazo equivalentes a 28 semanas que difícilmente podrían 
alterar la ganancia de peso esperada, la eficiencia económica se calculó considerando el sistema de producción como la tecnología, el alimento como el principal insumo, la ganancia de peso como el nivel de producción, ${ }^{1}$ el CMe como el costo promedio por $\mathrm{kg}$ de peso vivo y el $\mathrm{CMg}$ como el incremento en el costo por producir un $\mathrm{kg}$ adicional. Las expresiones matemáticas son:

\section{$C=$ Costo variable $=$ Costo del alimento}

$$
\begin{aligned}
C V M_{e} & =\frac{C \text { del alimento }}{\text { Ganancia de peso total del guajolote en pie }} \\
C V M_{g} & =\frac{\Delta C \text { del alimento }}{\Delta \text { Ganancia de peso total del guajolote en pie }}
\end{aligned}
$$

Donde, el costo del alimento se expresa en $\$ / \mathrm{kg}$ y la ganancia de peso en kg. Dada la relación entre $C T, C M e$ y $C M g$, a través de la representación gráfica e interpretación de la función de costos que se define como la relación entre el costo de adquirir los factores de producción y el nivel de producción obtenido (Pindyck y Rubinfeld, 2009), se determinó la relación entre el costo de alimento y la ganancia de peso para cada fase productiva de los guajolotes y se identificó el punto en el que $C M g=C M e$, es decir, el punto en el guajolote alcanza la ganancia máxima óptima de peso vivo y la unidad meleagrícola la eficiencia económica.

\section{3. Modelo estadístico}

A través de la revisión bibliográfica se identificó que para la meleagricultura no existen trabajos sobre la determinación de óptimos técnicos y económicos estimados a través de modelos econométricos no lineales; debido a lo anterior, la metodología se basa en trabajos que estiman dichos modelos para la producción ovina, bovina y porcina.

Para determinar los óptimos técnico y económico en corderos pelibuey engordados en corral en el Estado de México, Rebollar et al. (2008a) estimaron funciones de producción cuadráticas a través de modelos de regresión no lineales. Los óptimos técnicos para alimentación y número de semanas fueron 107.40 y $34.3 \mathrm{~kg}$, con ganancias de $718.29 \mathrm{y}$ 1147.61 pesos, y los óptimos económicos de 95.1 y 34.26 kg,

1. Ante la heterogeneidad de la información no fue posible considerar otros costos variables (mano de obra directa, bacterinas, vacunas, antibióticos) ni costos fijos (mano de obra indirecta, costo de las parvadas de pavipollos, materiales y suministros, activos fijos -depreciación-, servicios básicos, comercialización). con ganancias de 728.75 y 1148.13 pesos respectivamente. En el mismo año, Rebollar et al. (2008b) estimaron una función de producción cuadrática con rendimientos decrecientes para estimar los óptimos técnico y económico de una granja porcícola, la cual se ubica en Temascaltepec, Estado de México, que opera bajo sistema de producción semitecnificada y de la que se obtiene para la piara un óptimo técnico de 162.17 $\mathrm{kg}$ con 74 unidades de alimento, un óptimo económico de $142.77 \mathrm{~kg}$ con 49 unidades y un precio de venta de cerdo en pie en el mercado fue de $\$ 16.00 / \mathrm{kg}$.

Rebollar et al. (2011) estimaron dos funciones de producción cúbicas con rendimientos marginales decrecientes para determinar los óptimos para 100 novillos Bos taurus y Bos indicus con un peso vivo inicial de $290 \pm 15 \mathrm{~kg}$, de 21 a 24 meses de edad, engordados durante 93 días en el sur del Estado de México. En ambas funciones consideraron como variable dependiente la ganancia de peso y como variables independientes el consumo de alimento y el tiempo, para la primera y segunda funciones respectivamente. Encontraron que el punto óptimo de venta y la máxima ganancia se obtiene con la segunda función a un peso de $460.21 \mathrm{~kg}$ y un periodo de engorda de 77.21 días.

En este tenor, Rebollar et al. (2014) determinaron el peso óptimo de venta de cerdo en canal para dos regiones del Estado de México en función del rendimiento en cortes primarios, secundarios o terciarios. La estimación de tres modelos estadísticos no lineales ajustados a funciones de producción con rendimiento decrecientes arrojó que no existe una relación directa entre el peso de la canal y la ganancia de peso, dado que los ingresos por concepto de venta de cortes secundarios derivados de canales con un peso menor es mayor a los obtenidos por otro tipo de cortes, aun cuando las canales sean de mayor peso.

A partir de lo anterior, se plantearon y estimaron tres modelos para determinar los óptimos técnico y económico de la producción de guajolotes obtenida bajo sistemas de traspatio, semitecnificados y tecnificados en el Estado de México durante las 28 semanas de engorda. El modelo base para la estimación en los tres sistemas fue:

$V=\beta_{0}+\beta_{1} A+\beta_{2} A^{2}+\varepsilon$

Donde $V$ es la variable dependiente equivalente al peso vivo del guajolote expresado en $\mathrm{kg}, A$ la variable independiente asemejada al consumo de alimento referido en $\mathrm{kg} /$ semana y $\beta$ el coeficiente de regresión. Todos los datos para $i=0,1,2$. Una vez estimados los modelos, se calcularon los óptimos para cada sistema aplicando las siguientes expresiones matemáticas: 
Óptimo técnico $=P M g=\frac{\delta V}{\delta A}$

Óptimo económico $=P M g=\frac{P x}{P y}$

Donde, $P x$ es el precio del alimento y $P y$ el precio de venta del guajolote en pie, expresados en $\$ / \mathrm{kg}$. A partir de los resultados se graficaron las funciones de producción e identificaron los puntos donde se alcanzan los óptimos. ${ }^{2}$

\section{Resultados}

\section{1. Análisis diferencial}

A través del análisis de la producción de guajolote en el Estado de México se encontró evidencia de que esta actividad económica se realiza en unidades meleagrícolas que operan bajo tres sistemas de producción: traspatio, semitecnificados y tecnificados, resultados que coinciden con lo reportado por Díaz (1975) y Jerez et al. (1994), quienes refieren que la meleagricultura se realiza bajo tres diferentes escalas: a) grandes unidades de producción que operan bajo sistemas tecnificados, b) pequeños o medianos meleagricultores que basan su producción en sistemas semitecnificados y c) microproductores que crían los guajolotes con métodos tradicionales en traspatio.

Por otro lado, el análisis diferencial de las unidades meleagrícolas arrojó que la infraestructura, equipo, parvadas, alimentación y capital humano son los factores que determinan la diferencia entre los tres sistemas de producción. De acuerdo con la tabla 1, las unidades de traspatio no generan las condiciones idóneas para que esta actividad se lleve a cabo, pues a pesar de que el origen de los guajolotes contrarresta los efectos de las condiciones bajo las que son producidos, el poco control zoosanitario afecta la eficiencia técnico-económica. En contraste, la disponibilidad y uso

Tabla 1. Información general de las zonas de estudio.

\begin{tabular}{|c|c|c|c|}
\hline \multirow{2}{*}{ Factor } & \multicolumn{3}{|c|}{ Sistema de producción } \\
\hline & Traspatio & Semitecnificado & Tecnificado \\
\hline $\begin{array}{l}\text { Infraestructura y } \\
\text { equipo }\end{array}$ & $\begin{array}{l}\text { Gallineros tradicionales en la parte trasera de las } \\
\text { casas-habitación adaptados con perchas para descan- } \\
\text { so de las aves y nidos para la postura de los huevos. } \\
\text { La infraestructura de los gallineros es de ladrillo o } \\
\text { de marcos de madera forrados con tela de gallinero, } \\
\text { los techos de láminas de cartón, los comederos son } \\
\text { canaletas, bandejas de lámina o el piso del gallinero y } \\
\text { los bebederos recipientes de plástico. Sólo } 18 \% \text { de los } \\
\text { meleagricultores cuenta con criadoras caseras para el } \\
\text { manejo de las parvadas. }\end{array}$ & $\begin{array}{l}\text { Obsoleta y limitada tecnología. Inter- } \\
\text { media entre el sistema de traspatio y } \\
\text { el tecnificado. }\end{array}$ & $\begin{array}{l}\text { Casetas o naves bien ventiladas que } \\
\text { regulan la temperatura y evitan la } \\
\text { humedad del piso. Las paredes son } \\
\text { de tabique, los techos de lámina gal- } \\
\text { vanizada, las ventanas de tela para } \\
\text { gallinero y las cortinas de costales de } \\
\text { alimento; están equipadas con bebede- } \\
\text { ros y comederos automáticos. Para la } \\
\text { comercialización cuentan con un área } \\
\text { para el proceso de sacrificio y corte, y, } \\
\text { si es el caso, para el ahumado. }\end{array}$ \\
\hline Parvadas & $\begin{array}{l}\text { Conformadas por no más de } 10 \text { guajolotes: hembras, } \\
20 \% \text {; machos } 28 \% \text {; pavipollos } 52 \% \text {. La mayoría son } \\
\text { criollos de plumaje negro o bronceado, de talla menor } \\
\text { a la del guajolote mejorado y de alta rusticidad y resis- } \\
\text { tencia a climas adversos. }\end{array}$ & $\begin{array}{l}\text { Formadas por 50-70 guajolotes criollos } \\
\text { y líneas comerciales. }\end{array}$ & $\begin{array}{l}\text { La mayoría de las parvadas son homo- } \\
\text { géneas y están integradas por 100-150 } \\
\text { guajolotes genéticamente mejorados de } \\
\text { raza diamante blanco, procedentes de } \\
\text { EE. UU. y Canadá. }\end{array}$ \\
\hline Alimentación & $\begin{array}{l}\text { De manera general, la dieta está conformada por: } \\
\text { sobrantes de cocina } 32 \% \text {, pastoreo } 16 \% \text {, maíz } 14 \% \text {, } \\
\text { tortilla } 12 \% \text {, alimento comercial } 10 \% \text {, sema } 9 \% \text { y alfalfa } \\
7 \% \text {. A fin de reducir la mortalidad de los pavipollos y } \\
\text { asegurar su rápido crecimiento, ocasionalmente se les } \\
\text { da alimento comercial combinado con la alimentación } \\
\text { que se les otorga a los guajolotes adultos. }\end{array}$ & $\begin{array}{l}\text { Alimentación basada en maíz, sorgo y } \\
\text { alimento comercial. }\end{array}$ & $\begin{array}{l}\text { A las parvadas se les alimenta con } \\
\text { alimento comercial que cubre sus } \\
\text { requerimientos nutricionales durante } \\
\text { cada fase productiva: iniciación, creci- } \\
\text { miento, engorda y finalización. }\end{array}$ \\
\hline $\begin{array}{l}\text { Capital humano } \\
\text { (mano de obra) }\end{array}$ & $\begin{array}{l}\text { Familiar, representada por amas de casa que realizan } \\
\text { la limpieza de gallineros, jefes de familia que sacrifican } \\
\text { y venden los guajolotes e hijos que alimentan a las } \\
\text { parvadas. Tiempo promedio diario (en horas) destina- } \\
\text { do para realizar estas actividades: } 2 \text { a } 4(63 \%) \text {, } 4 \text { a } 8 \\
(21 \%) \text { y } 8(16 \%) \text {. }\end{array}$ & $\begin{array}{l}\text { No utilizan mano de obra familiar y, en } \\
\text { la mayoría de los casos, el personal no } \\
\text { está capacitado. }\end{array}$ & $\begin{array}{l}\text { Mano de obra directa e indirecta capa- } \\
\text { citada y calificada acorde al área que } \\
\text { pertenece y actividad que desempeña: } \\
\text { producción y sacrificio o administra- } \\
\text { ción y comercialización. }\end{array}$ \\
\hline
\end{tabular}

Fuente: elaboración propia con información obtenida a través de encuestas aplicadas en las unidades meleagrícolas visitadas.

2. En la estimación del modelo estadístico se utilizó el paquete computacional Eviews 7. 
adecuado de los factores de producción en las unidades tecnificadas favorecen la ejecución de estrictos controles sanitarios y de calidad en la producción y sacrificio del guajolote, así como en el empacado, congelación y transporte de la carne de esta especie.

El análisis espacial indica que la distribución geográfica de las unidades meleagrícolas incide en la participación que tienen los tres sistemas en la producción estatal y en el mercado. La distribución en todo el territorio mexiquense de la meleagricultura de traspatio y su desempeño durante todo el año como actividad principal o intercalada con otras actividades agropecuarias y no agropecuarias ha permitido generar $75 \%$ de la producción, mientras que el reducido número de unidades meleagrícolas semitecnificadas y tecnificadas y su dispersa distribución espacial ha propiciado que contribuye con 20 unidades $5 \%$ de la producción estatal respectivamente.

A diferencia del mercadeo anterior, las unidades tecnificadas le otorgan valor agregado al guajolote en pie mediante el sacrificio para ofertarlo entero (fresco, congelado, ahumado u horneado) o en piezas (pechuga y pierna-muslo). A través de canales de comercialización bien definidos cubren mercados mayoristas y minoristas de carne fresca, e industrias de carnes frías y embutidos ubicadas en centros urbanos. Asimismo, algunos meleagricultores que realizan desplume en seco durante el proceso de sacrificio cubren nichos de mercado específico y selectivo de Toluca y de la Ciudad México, donde obtienen precios superiores al precio de referencia de mercado.

Los resultados del análisis diferencial meleagrícola de traspatio coinciden con los reportados por Camacho-Escobar et al. (2006), Mallia (1998) y Rodríguez et al. (1996), quienes concluyen que esta actividad carece de buenas prácticas técnico-productivas y comerciales que la hacen poco o nada rentable, pero que representa una oportunidad para transformar los recursos locales en guajolote en pie equivalente a carne y huevo y de conservar la actividad como una tradición sociocultural. Para las unidades meleagrícolas semitecnificadas y tecnificadas no se encontró evidencia empírica para contrastar los resultados obtenidos; sin embargo, se pudo comprobar que las segundas son las únicas que implementan sistemas de producción encaminados a cubrir los requerimientos nutricionales de los guajolotes propuestos por Guibon et al. (1999).

\section{2. Análisis de las variables}

El análisis se llevó a cabo para la engorda de 2011 y el periodo fue de 28 semanas. La principal variable utilizada para determinar la eficiencia técnica fue la ganancia de peso vivo del guajolote, que se obtuvo a partir del consumo de alimento y la conversión alimenticia. Durante el periodo de engorda, la ganancia de peso máximo en los guajolotes en las unidades meleagrícolas fue la siguiente: traspatio $7.83 \mathrm{~kg}$, semitecnificadas $11.35 \mathrm{~kg}$ y tecnificadas $18.70 \mathrm{~kg}$ (cuadro 2). La diferencia de peso indica que para producir un kilogramo de peso vivo en el primer sistema se requirió un consumo semanal de 1.89 $\mathrm{kg}$ de alimento, en el segundo $3.45 \mathrm{y}$ en el tercero 2.2.

Para la eficiencia económica, las variables analizadas fueron el costo del alimento y el precio de comercialización del guajolote en pie. De acuerdo con el cuadro 3, la relación entre las dos variables para las tres unidades meleagrícolas es positiva: traspatio 2.5, semitecnificada 6.9, tecnificada 14. Sin embargo, a esta utilidad bruta habría que restarle otros costos variables y los costos fijos para obtener la utilidad neta. 


\section{3. Análisis estadístico de las funciones de producción}

Los valores obtenidos de los parámetros estadísticos de cada modelo confirmaron la bondad de ajuste de los mismos; empero, ante la presencia de autocorrelación positiva de primer orden en los tres casos determinada por el estadístico Durbin Watson (Dw) (Gujarati, 2007), se realizó una prueba general de autocorrelación con el estadístico BreuschGodfrey (LM). De acuerdo con los resultados de la prueba LM, se acepta la hipótesis nula de que no existe autocorrelación serial; por lo tanto, es válida la utilización de los modelos.

$\mathrm{El}$ análisis de los términos de error o residuales indica que las suposiciones asociadas con su distribución son aceptadas y, en relación con las ecuaciones de regresión o funciones de producción, se encontró que éstas se ajustan a las teorías económica y estadística, dado que los signos obtenidos fueron los esperados (cuadro 4).

La $F$ calculada en los modelos $(29.92,10.77$ y 20.55$)$ resultó altamente significativa (ANDEVA; $p<0.01$ ), lo que quiere decir que el número de veces que el cuadrado medio de la regresión contiene al cuadrado medio del error; además, al realizar la prueba para estimar el parámetro verdadero con un nivel de significancia de 95\%, se rechaza la hipótesis de que la variable independiente no se relaciona con el peso de los guajolotes. De manera similar, el coeficiente de determinación $R^{2}$, permitió detectar que $99.55,98.76$ y $99.34 \%$ de la variación en el peso de los guajolotes para cada sistema, lo explica el modelo de regresión (cuadro 4). Finalmente, cada estimación del parámetro (coeficiente asociado con la variable independiente) equivale a más del doble de su error estándar, lo cual puede corroborarse a través de los valores de la $t$ de student.

\section{4. Óptimos técnico y económico \\ 2. 4. 1.Óptimo técnico}

En las unidades meleagrícolas tecnificadas los guajolotes alcanzan el peso vivo óptimo entre la semana 22 y 23 con un consumo promedio de $63.3 \mathrm{~kg}$, en las semitecnificadas entre la 27 y 28 con $32.6 \mathrm{~kg}$ de alimento (cuadro 5), y como resultado de una dieta desbalanceada en traspatio los guajolotes no alcanzan el peso vivo óptimo.

En la gráfica 1 se muestra la relación entre el consumo de alimento y la ganancia de peso vivo del guajolote para unidades meleagrícolas tecnificadas y semitecnificadas. En la etapa I de la función de producción se observa que existe una relación directa como consecuencia de la transición de las fases productivas del

Gráfica 1. Sistemas meleagrícolas semitecnificados y tecnificados mexiquenses: función de producción óptima (2011).

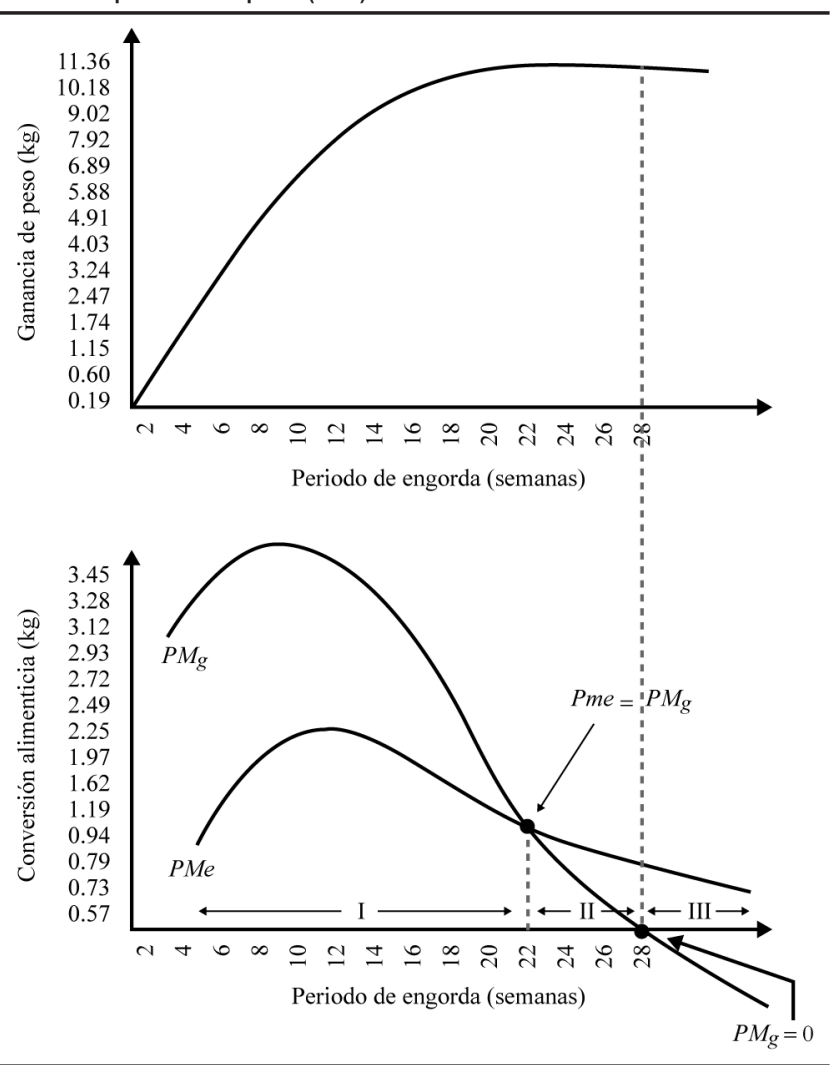

Fuente: elaboración propia con información del cuadro 1 , tabla 1 y cuadro 2.

\begin{tabular}{cccccc}
\hline Cuadro 4. & Parámetros estadísticos para la meleagricultura mexiquense (2011). \\
Sistema & \multicolumn{2}{c}{ Ecuaciones } & DW & LM \\
\hline Tecnificado & $V=0.516929465518+0.526060714425 * A$ & $0.00413656297573 * A^{\wedge} 2$ & \\
$t$ & $(4.518415)$ & $(3.557501)$ & $(-2.631085)$ & 0.5446 & 0.061 \\
$e e$ & $(0.0001)$ & $(0.0000)$ & $(0.0000)$ & & \\
Semi-tecnificado & $V=0.797592914805+0.402099001975 * A$ & $0.00361689980095 * A^{\wedge} 2$ & \\
$t$ & $(3.901129)$ & $(2.302673)$ & $(-2.156869)$ & 0.13490 .0546 \\
$e e$ & $(0.0006)$ & $(0,0000)$ & $(0.0000)$ & \\
Traspatio & $V=0.454954724939+0.438973990751 * A-0.00591973607141 * A^{\wedge} 2$ & \\
$t$ & $(4.962044)$ & $(2.507577)$ & $(-8.675479)$ & 0.12180 .0587 \\
$e e$ & $(0.0000)$ & $(0.0000)$ & $(0.0000)$ & \\
\hline
\end{tabular}

Nota: ee: errores estándar

Fuente: elaboración propia con información recopilada en unidades meleagrícolas.

Cuadro 5. Sistemas productivos meleagrícolas mexiquenses: óptimos técnicos (2011).

\begin{tabular}{lcccc}
\hline Óptimos & Tecnificado & Semitecnificado & Traspatio \\
\hline \multirow{2}{*}{ Técnico } & $A$ & 63.6 & 32.6 & 37.1 \\
& $V$ & 17.2 & 10.1 & 8.6 \\
\hline
\end{tabular}

Nota: $A$ = variable independiente asemejada al consumo de alimento referido en $\mathrm{kg} / \mathrm{semana}$; $V=$ variable dependiente equivalente al peso vivo del guajolote expresado en $\mathrm{kg}$.

Fuente: elaboración propia con resultados de los modelos estimados. 
guajolote, dado que de encontrarse en la fase de iniciación, pasa a la de crecimiento y llega a la de engorda (semanas 0 a 17). En la etapa II, el guajolote obtiene el peso máximo óptimo de $12 \mathrm{~kg}$. Por este motivo, la combinación insumoproducto es eficiente y la ganancia de peso proporcional al consumo de alimento; sin embargo, si al final de esta etapa (inicio de la etapa III, semana 28) no ha alcanzado el peso esperado, la unidad meleagrícola obtendrá rendimientos decrecientes porque a partir de ese momento el guajolote no aumentará su peso ante un incremento en el consumo de alimento, sino que por el contrario tenderá a bajar ante las diarreas producidas por el exceso de alimento consumido.

En el caso de las unidades de traspatio el desconocimiento sobre los requerimientos nutricionales de los guajolotes genera que la calidad y cantidad de alimento, así como la hora y número de veces que le den de comer a los guajolotes sea variado; por consecuencia, el consumo de alimento es alto y en lugar de transformarlo en peso vivo equivalente a carne se convierte en grasa y no se obtiene el óptimo técnico ni la eficiencia técnica.

\section{4. 2. Óptimo económico}

Dado que el costo del alimento registrado en las unidades meleagrícolas de traspatio únicamente se refiere al de los pavipollos en fase de iniciación, éste fue de 10 a 12 pesos, superior al registrado en las semitecnificadas (\$6.50) y tecnificadas (\$5.00). Por otro lado, la diferencia en los precios de venta del guajolote en pie arrojo un margen de $\$ 40.00 / \mathrm{kg}$ entre las unidades tecnificadas y de traspatio y de $\$ 25.00 / \mathrm{kg}$ entre tecnificadas y semitecnificadas. Ante estas diferencias, las unidades tecnificadas alcanzan la eficiencia económica en el momento en que el guajolote llega a un peso vivo de $16.9 \mathrm{~kg}$ con un consumo de $54.5 \mathrm{~kg}$, las semitecnificadas a los $7.3 \mathrm{~kg}$ y un consumo de $19.6 \mathrm{~kg}$ y las de traspatio a un peso de $5.8 \mathrm{~kg}$ y un consumo de $15.4 \mathrm{~kg}$ (cuadro 6); no obstante, en las últimas no se tiene un control sobre el costo de la alimentación de las parvadas por ser prácticamente a base de desperdicios agrícolas o sobrantes de cocina, por lo cual los márgenes de ganancia se reducen.

Ante los resultados anteriores, en los tres tipos de unidades meleagrícolas se cumple el supuesto teórico de que el óptimo económico se encuentra por debajo del técnico, aunque con un marcado contraste entre $P M e$ y $P M g$.

Cuadro 6. Óptimo técnico en tres sistemas productivos de guajolotes (2011).

\begin{tabular}{lcccr}
\hline Óptimos & Tecnificado & Semitecnificado & Traspatio \\
\hline \multirow{2}{*}{ Económico } & $A$ & 54.5 & 19.6 & 15.4 \\
& $V$ & 16.9 & 7.3 & 5.8 \\
\hline Fuente: elaboración propia con resultados de los modelos estimados. & \\
\hline
\end{tabular}

\section{Conclusiones}

A través del análisis diferencial de la producción de guajolotes en el Estado de México se encontró evidencia de que esta actividad económica se desarrolla bajo sistemas de producción familiar, tradicional o de traspatio, semitecnificados y tecnificados. La disponibilidad y buena administración de los factores de producción han permitido a las unidades meleagrícolas tecnificadas obtener un alto grado de especialización y nivel de integración. Además, han logrado una mayor eficiencia técnico-económica que se ve reflejada en la ganancia de peso vivo que alcanzan los guajolotes durante el periodo de engorda, en el valor agregado que le otorgan durante el proceso de sacrificio y en los nichos de mercado que cubren. En contraste, la meleagricultura de traspatio enfrenta problemas técnico-productivos, económicos y comerciales que no le permiten generar condiciones adecuadas para que los guajolotes obtengan la ganancia de peso vivo óptimo durante el periodo de engorda, a pesar de ser una actividad que se ha conservado como una tradición socio-cultural por representar una oportunidad de transformar los recursos locales en guajolote en pie y generar ingresos para un gran número de familias.

Los hallazgos presentados llevan a suponer que la meleagricultura mexiquense seguirá orientándose más hacia los sistemas de traspatio; sin embargo, es recomendable que estas unidades productivas y las semitecnificadas implementen estrategias técnico-productivas y comerciales encaminadas a mejorar y optimizar los factores de producción a fin de incrementar su eficiencia técnico-económica.

\section{Análisis prospectivo}

La evidencia empírica indica que la meleagricultura mexiquense se realiza bajo un escenario poco competitivo dada la diferencia entre los sistemas productivos. Por este motivo, es necesario plantear opciones y construir escenarios estratégicos encaminados a visualizar la tendencia prospectiva. El patrón histórico indica que, a pesar de las características técnico-productivas y económicas de las unidades meleagrícolas que operan bajo sistemas de traspatio consideradas como debilidades, esta actividad ha representado una oportunidad de convertir los recursos disponibles o (factores de producción) en carne, intercalar la meleagricultura con otras actividades económicas, utilizar mano de obra familiar y generar ingresos. Aunado a lo anterior, dada la demanda de guajolote en mercados 
locales durante festividades religiosas, familiares o de fin de año, los cambios en las tendencias de consumo no han tenido efectos sobre esta actividad, por lo que se espera que prevalezca.

A diferencia de las unidades meleagrícolas de traspatio, las semitecnificadas han sido altamente sensibles a cambios en las tendencias del consumo y precios de los insumos, dado que cubren nichos de mercado muy específicos y generan altos costos de producción que las hacen poco rentables. Ante esto, tendrían que implementar estratégicas técnico-productivas y económicas encaminadas a incrementar su grado de especialización, nivel de integración y eficiencia; de lo contrario, corren el riesgo de desaparecer ante situaciones adversas. Finalmente se tiene que el grado de especialización y nivel de integración de las unidades meleagrícolas con sistemas tecnificados han minimizado su costos de producción y generado, a través del valor agregado durante la producción y sacrificio, productos diferenciados por su calidad y precio que han sido competitivos en el mercado, por lo que difícilmente enfrentarán situaciones complicadas que las pongan en riesgo.

Derivado de lo expuesto y de la distribución espacial de la producción, se puede esperar que los municipios del sur del estado sigan siendo los que tengan menor participación a nivel estatal, en contraste con los del centro y norte, puesto que en estas dos regiones se encuentran Toluca, Atlacomulco y Zumpango, que han destacado por generar de manera conjunta casi $70 \%$ de la meleagricultura mexiquense.

\section{Referencias}

Camacho-Escobar, M., Lira-Torres, I., Ramírez-Cancino, L., López-Pozos, R. y Arcos-García, J. (2006). La avicultura de traspatio en la Costa de Oaxaca, México. Ciencia y Mar, 10(28): 3-11.

Díaz, G. (1975). Programa Nacional de Meleagricultura. Memoria Primera. Edición Anual. México: Dirección General de Avicultura y Especies Menores.

Financiera Rural (2012). Monografía del guajolote o pavo. Consultado en noviembre de 2012. Disponible en http://www. financierarural.gob.mx/informacionsectorrural/Documents/Monografias/ MonografiaGuajolote(dic\%2010)vf.pdf

Guibon, A., Sibbald, A. y Thomas, C. (1999). Improved sustainability in livestock systems, a challenges for animal production science. Livestock Production Science, 61:107-110.

Gujarati (2007). Econometría (4a ed.). McGrawHill.

INEGI (Instituto Nacional de Estadística y Geografía) (2007). Censo Agrícola, Ganadero y Forestal. Consultado en septiembre de 2012. Disponible en http://www.inegi. org.mx/est/contenidos/proyectos/Agro/
ca2007/Resultados_Agricola/.

Jerez, M., Herrera, J. y Vásquez, M. (1994). La gallina criolla en los valles centrales de Oaxaca. Oaxaca: ITAO-CIGA.

Mallia, G. (1998). Indigenous domestic turkeys of Oaxaca and Quintana Roo, Mexico. Animal Genetic Resourses Information, 23: 68-78.

Pindcky, R. y Rubinfeld, D. (2009). Econometría (7a ed.). Pearson-Prentice Hall.

Rebollar, S., Hernández, J., Rojo, R., González, F., Mejía, D. y Cardoso, J. (2008a). Óptimos económicos en corderos pelibuey engordados en corral. Universidady Ciencia, 24(1): 67-73.

Rebollar, S., Gómez, G., Hernández, J., Rojo, R., González, F. y Avilés, F. (2008b). Determinación del óptimo técnico y económico en una granja porcina en Temascaltepec, Estado de México. CIENCIA ergo-sum, 14(3): 255-262.

Rebollar, S., Posadas, R., Hernández, J., González, F., Guzmán, E. y Rojo, R. (2011). Technical and economics optimal in feedlot cattle. Tropical and Subtropical Agroecosystems, 14(2):413-420.

Rebollar, S., Gómez, G., Callejas, N., Guzmán, E. y Hernández, J. (2014). Óptimos técnicos y económicos en cortes de carne de cerdo en dos regiones de México. Agronomía Mesoamericana, 25(1): 161-168.

Rodríguez, J., Allaway, C., Wassink, G., Segura, C. y Rivera, T. (1996). Estudio de la avicultura de traspatio en el municipio de Dzununcán, Yucatán. Veterinaria México, 27(3): 215-219.

Sagarpa (Secretaría de Agricultura, Ganadería, Desarrollo Rural, Pesca y Alimentación) (2012). Estadísticas sobre el sector ganadero. Producción de guajolote y carne en canal en el Estado de México. Consultado en marzo de 2013. Disponible en http://www.siap.gob.mx.

Siacon (Sistema de Información Agroalimentaria de Consulta) (2013). Programa Informático con base de datos agrícolas, pecuarios y pesqueros. Volumen, precios medios rurales y valor de la producción de guajolote en pie y de carne en canal (2000-2012). México.

SIAP (Servicio de Información Agroalimentaria y Pesquera) (2012). Producción y peso de guajolote en pie y carne en canal a nivel municipal. Consultado en diciembre de 2012. Disponible en http://infosiap.siap.gob.mx/ anpecuario_siap/ProduccionMunicipio.do 